\title{
SOME NECESSARY CONDITIONS FOR CONVERGENCE OF THE GBDF METHODS
}

\author{
MOHAMED BIN SULEIMAN
}

\begin{abstract}
The Generalized Backward Differentiation methods for solving stiff higher-order ordinary differential equations are described. The convergence, zero stability and consistency of these methods are defined. Next, the zero stability and consistency conditions necessary for convergence are proven. The order for which the methods are zero stable is also determined.
\end{abstract}

\section{INTRODUCTION}

We shall consider the numerical solution of higher-order ordinary differential equations (ODEs) given by

$$
y_{i}^{\left(d_{i}\right)}=f_{i}(x, Y), \quad i=1,2, \ldots, s, \quad Y(a)=\eta,
$$

where the $i$ th equation is of order $d_{i}$ and

$$
\begin{aligned}
Y^{T} & =\left(y_{1}, \ldots, y_{1}^{\left(d_{1}-1\right)}, \ldots, y_{s}, \ldots, y_{s}^{\left(d_{s}-1\right)}\right), \\
\eta^{T} & =\left(\eta_{10}, \ldots, \eta_{1, d_{1}-1}, \ldots, \eta_{s, 0}, \ldots, \eta_{s, d_{s}-1}\right) .
\end{aligned}
$$

Many of these problems which arise naturally in physical situations are stiff. The problem in (1.1) is considered stiff whenever the corresponding first-order system is stiff. A method proposed by Krogh [5] for solving (1.1) directly is to interpolate back values of $y_{i}^{\left(d_{i}-j_{i}\right)}, j_{i}=0,1, \ldots, d_{i}$, by polynomials $P_{k_{i}}(x)$ of degree $k_{i}$, differentiating or integrating as the case may be to obtain the other derivatives, and then equating them using (1.1). The integer $j_{i}$ is termed the stiffness capability index. The method when $j_{i}=0$ is called the Direct Integration Method (DI) and is associated with solving nonstiff problems. The convergence of the variable-order and variable-stepsize version of this method is proven in [6]. The stability of this method for second-order ODEs is discussed in [3] and [2].

The case $j_{i}>0$ is associated with stiff problems and is called the Generalized Backward Differentiation (GBDF) method. An attempt has been made in [7] to develop a strategy for the correct choice of $j$, the stiffness capability index, for a single second-order ODE.

In this paper we will discuss some necessary conditions for convergence of the constant-stepsize GBDF method. These conditions are those that are normally associated with a multistep method, namely, zero stability and consistency. The

Received by the editor May 11, 1989 and, in revised form, April 16, 1990 and April 30, 1991. 1991 Mathematics Subject Classification. Primary 65L05, 65L20. 
coefficients of the GBDF method for various $j$ will also be generated, and the order for which the method is zero stable will be determined. Prior to the above discussions, some numerical results will be given which give some indication of how a higher-order ODE should be solved by the GBDF method. These results motivate us to look at the theory behind the method.

\section{SOME NUMERICAL RESULTS USING THE GBDF METHOD}

The need for a theoretical study of the GBDF method arises from some recent results on solving higher-order ODEs directly by multistep methods. These results, given in [7] and [8], show that in many problems the direct method is more desirable than reduction to first-order systems. The prime reason for this is that, for the solution of (1.1), we require an interpolation polynomial with $s$-dimensional vector coefficients, whereas transformation to a first-order system requires higher-dimensional $\left(s^{*}\right.$-dimensional, where $\left.s^{*}=\sum_{i=1}^{s} d_{i}\right)$ vector coefficients. This accounts for a considerable saving in storage and overhead. The work in [8] deals with the DI method, the case $j_{i}=0$, for solving nonstiff problems. In [7] results are given for a single stiff second-order ODE. Also given in [7] is a discussion of the correct choice of $j$ for a second-order ODE. For this, the absolute stability regions of the direct method for cases $j=0,1$ and 2 of the simple, scalar test problem

$$
y^{\prime \prime}=\theta y^{\prime}+\mu y
$$

are investigated.

It has been noted that for the same step number $k$, the method with $j=2$ has better stability than the one with $j=1$, while the method with $j=1$ has better stability than the one with $j=0$. For the case $j=2$, the method is stiffly stable. The best approach to solve problem (2.1) is to start with $j=0$ and then switch to $j=1$ when instability occurs with $j=0$, and subsequently to $j=2$ when instability occurs again with $j=1$. A similar generalization can be made for other higher-order ODEs. An indication of instability in a variableorder and variable-stepsize multistep code is frequent step failures at low order, viz., $k \leq 4$ for $j=0$ and $k=j+1$ for $j>0$. For the problem in (2.1) other tests which ascertain instability were developed before $j$ is increased, cf. [7].

Raising $j$ to $j+1$ at the point of instability increases the efficiency of the method. First, it ensures better stability, which consequently allows for the use of larger stepsizes within the required accuracy. Second, it allows the use of the minimum value of $j$, as long as stability permits it. This implies that, whenever accuracy rather than stability is determining the stepsize, we are using the GBDF method whose derivatives have local errors with the smallest magnitudes. This results in better accuracy or the use of larger stepsizes or both. Further, it allows the use of fixed point iteration at $j=0$, which is computationally cheaper than Newton's iteration.

For the two problems below we present numerical results, using our variableorder and variable-stepsize GBDF code. For the second-order problem, we incorporate the tests for increasing $j$ developed in [7]. For the third-order problem we check indications of instability, that is, step failures at low order, to increase $j$. We increase $j=0$ to $j=1$ if $k \leq 4$, and $j$ to $j+1$ if $k=j+1$. This illustrates the capability of the GBDF method. Investigations are still being carried out with respect to other tests for increasing $j$. In parentheses 
for the first two tables are the results when the problems are reduced to firstorder systems and solved using the standard BDF method. The notations are self-explanatory, except for

TOL: tolerance used,

$[j ;(x, h)]$ : The stiffness capability index is increased from $j-1$ to $j$ at the point $x$ and when the stepsize is $h$.

Throughout, a number of the form $4.5(-3)$ will mean $4.5 \times 10^{-3}$.

\section{Problem 1.}

$$
\begin{aligned}
& y^{\prime \prime}+300 x y^{\prime}+300 y=900 x^{2}+302 \\
& y(0)=2, \quad y^{\prime}(0)=0, \quad 0 \leq x \leq 30 .
\end{aligned}
$$

Solution. $1+x^{2}+e^{-150 x^{2}}$.

This problem is adapted from the stiff problem given in Varah [9].

TABLE 1. Results for Problem 1

\begin{tabular}{|cccccc|}
\hline $\log _{10}$ TOL & $\begin{array}{c}\text { Total } \\
\text { Number } \\
\text { of Steps }\end{array}$ & $\begin{array}{c}\text { Total } \\
\text { Failures } \\
\text { Failus }\end{array}$ & $\begin{array}{c}\text { Jacobian } \\
\text { Evaluations }\end{array}$ & $\begin{array}{c}\text { Maximum } \\
\text { Error } \\
\operatorname{Max}_{i}\left|y\left(x_{i}\right)-y_{i}\right|\end{array}$ & {$[j ;(x, h)]$} \\
\hline-2 & 54 & 10 & 7 & $7.0(-2)$ & {$[1 ;(4.0(-1), 8.6(-3))]$} \\
& $(55)$ & $(27)$ & $(19)$ & $(2.9(-2))$ & \\
& & & & & \\
-4 & 57 & 6 & 9 & $1.3(-3)$ & {$[1 ;(4.1(-1), 1.2(-2))]$} \\
& $(79)$ & $(6)$ & $(29)$ & $(5.6(-5))$ & \\
& & & & & \\
-6 & 71 & 9 & 11 & $1.7(-5)$ & {$[1 ;(3.8(-1), 1.6(-2))]$} \\
& $(144)$ & $(9)$ & $(39)$ & $(2.3(-6))$ & \\
\hline
\end{tabular}

\section{Problem 2.}

$$
\begin{array}{r}
y^{\prime \prime \prime}=\left(10^{5}+10^{3}+10\right) y^{\prime \prime}-\left(10^{8}+10^{6}+10^{4}\right) y^{\prime}-10^{9} y+10^{8} \\
y(0)=3.1, \quad y^{\prime}(0)=-\left(10^{5}+10^{3}+10\right), \quad y^{\prime \prime}(0)=\left(10^{10}+10^{6}+10^{2}\right) \\
0 \leq x \leq 50 .
\end{array}
$$

Solution. $0.1+\exp \left(-10^{5} x\right)+\exp \left(-10^{3} x\right)+\exp (-10 x)$.

For the results given, the BDF implementation is the one which is obtained within the GBDF implementation, after applying it to the transformed firstorder system, that is, fixing $j=1$ throughout for each equation in the firstorder system. Notice that the computed solution using the BDF method is far more accurate than the tolerance required, especially on the second problem. This is because in the reduction to a first-order system, the local errors of all the derivatives are controlled, whereas in the GBDF method, only the local error of $y^{(d-1)}$ for $j=0$ or $y^{(d-j)}$ for $j>0$ is controlled. Therefore, in the BDF case, the error control strategy is more restrictive and hence resulting in the larger number of steps. Further, the cost per step is generally higher for the BDF 
TABLe 2. Results for Problem 2

\begin{tabular}{|c|c|c|c|c|c|}
\hline $\log _{10} \mathrm{TOL}$ & $\begin{array}{l}\text { Total } \\
\text { Number } \\
\text { of Steps }\end{array}$ & $\begin{array}{l}\text { Total } \\
\text { Step } \\
\text { Failures }\end{array}$ & $\begin{array}{l}\text { Jacobian } \\
\text { Evaluations }\end{array}$ & $\begin{array}{c}\text { Maximum } \\
\text { Error } \\
\operatorname{Max}_{i}\left|y\left(x_{i}\right)-y_{i}\right|\end{array}$ & {$[j ;(x, h)]$} \\
\hline \multirow[t]{4}{*}{-2} & 123 & 7 & 6 & $6.5(-2)$ & {$[1 ;(2.7(-4), 1.7(-5))]$} \\
\hline & & & & & {$[2 ;(1.1(-2), 1.7(-3))]$} \\
\hline & & & & & {$[3 ;(3.6(0), 4.4(-2))]$} \\
\hline & (786) & 37 & $(101)$ & $(1.0(-5))$ & \\
\hline \multirow[t]{4}{*}{-4} & 184 & 11 & 17 & $4.4(-4)$ & {$[1 ;(2.7(-4), 1.3(-5))]$} \\
\hline & & & & & {$[2 ;(5.7(-1), 9.0(-3))]$} \\
\hline & & & & & {$[3 ;(3.0(0), 3.8(-1))]$} \\
\hline & $(1537)$ & (14) & (94) & $(1.8(-6))$ & \\
\hline \multirow[t]{4}{*}{-6} & 251 & 7 & 15 & $9.9(-6)$ & {$[1 ;(7.2(-4), 1.0(-5))]$} \\
\hline & & & & & {$[2 ;(4.5(-2), 4.6(-3))]$} \\
\hline & & & & & {$[3 ;(9.5(0), 9.2(-1))]$} \\
\hline & (3159) & (16) & (114) & $(2.8(-8))$ & \\
\hline
\end{tabular}

method compared to the GBDF case, as the former involves higher-dimensional vector coefficients for the polynomial interpolation and higher overhead for the related divided differences and the control of the local errors of all the derivatives. Thus, the direct method, a combination of the DI and the GBDF methods, shows an advantage over the BDF method.

TABle 3. Results restricting $j$ up to 1 and 2 for Problem 2. The first row for each tolerance gives the result for $j=1$ and the second row in parentheses for $j=2$. The values of $(x, h)$ at which $j$ is increased to 1 and 2 are the same as in Table 2

\begin{tabular}{|ccccc|}
\hline $\log _{10}$ TOL & $\begin{array}{c}\text { Total } \\
\text { Number } \\
\text { of Steps }\end{array}$ & $\begin{array}{c}\text { Total } \\
\text { Step } \\
\text { Failures }\end{array}$ & $\begin{array}{c}\text { Jacobian } \\
\text { Evaluations }\end{array}$ & $\begin{array}{c}\text { Maximum } \\
\text { Error } \\
\operatorname{Max}_{i}\left|y\left(x_{i}\right)-y_{i}\right|\end{array}$ \\
\hline-2 & 827 & 82 & 137 & $6.5(-2)$ \\
& $(146)$ & $(20)$ & $(14)$ & $(6.5(-2))$ \\
-4 & 796 & 64 & 87 & $4.4(-4)$ \\
& $(197)$ & $(12)$ & $(22)$ & $(4.4(-4))$ \\
& & & & \\
& 982 & 83 & 109 & $9.9(-6)$ \\
& $(299)$ & $(18)$ & $(16)$ & $(9.9(-6))$ \\
\hline
\end{tabular}


For Problem 1, it suffices to solve the problem with $j$ up to 1 . The stepsize does not increase sufficiently to cause instability for $j=1$. This is due to the stepsize constraint for accuracy of the steady state term $x^{2}$. For Problem 2, the index $j$ may be increased gradually from 0 to 3 .

Table 3 shows the result for Problem 2 when using the direct method and restricting first $j$ to 1 , and then $j$ to 2 . Restricting $j$ to 1 needs far too many steps to finish the integration and frequent step failures, indicating the restriction of stepsize increase due to stability. Restricting $j$ to 2 shows a far better result compared to restricting $j$ to 1 in terms of total steps, but most efficient is still the gradual increase of $j$ to 3 . Note that the maximum global errors for the direct method in Tables 2 and 3 indicate that they occur just prior to $j$ being increased to 1 . These results show the merit of the GBDF method and the way it should be implemented.

\section{THE GBDF METHOD}

For ease of notation, and without loss of generality, a single $d$ th-order ODE will be considered,

$$
y^{(d)}=f(x, Y), \quad Y(a)=\eta,
$$

where $Y^{T}=\left(y, y^{\prime}, \ldots, y^{(d-1)}\right)$ and $\eta^{T}=\left(\eta_{0}, \eta_{0}^{\prime}, \ldots, \eta_{0}^{(d-1)}\right)$. Let $P_{k}(x)$ be the $k$ th-degree polynomial which interpolates the set $\left\{\left(x_{n+1-r}, y_{n+1-r}^{(d-j)}\right)\right\}_{r=0}^{k}$, the equally spaced back values of steplength $h$ of the derivative $y^{(d-j)}$, and is given by

$$
P_{k}(x)=\sum_{i=0}^{k} L_{i}(x) y_{n+1-i}^{(d-j)}, \quad \text { where } L_{i}(x)=\prod_{\substack{j=0 \\ j \neq i}}^{k} \frac{\left(x-x_{n+1-j}\right)}{\left(x_{n+1-i}-x_{n+1-j}\right)}
$$

The other derivatives at $x_{n+1}$ are obtained by differentiating or integrating $P_{k}(x)$ successively, as the case may be, giving

$$
y_{n+1}^{(r)}=\left\{\begin{array}{c}
y_{n}^{(r)}+h y_{n}^{(r+1)}+\cdots+\frac{h^{d-j-r-1}}{(d-j-r-1) !} y_{n}^{(d-j-1)} \\
+h^{d-j-r} \sum_{i=0}^{k} \alpha_{i, r} y_{n+1-i}^{(d-j)}, \quad r=0, \ldots, d-j-1, \\
\frac{1}{h^{r-(d-j)}} \sum_{i=0}^{k} \alpha_{i, r} y_{n+1-i}^{(d-j)}, \quad r=d-j+1, \ldots, d-1,
\end{array}\right.
$$

where

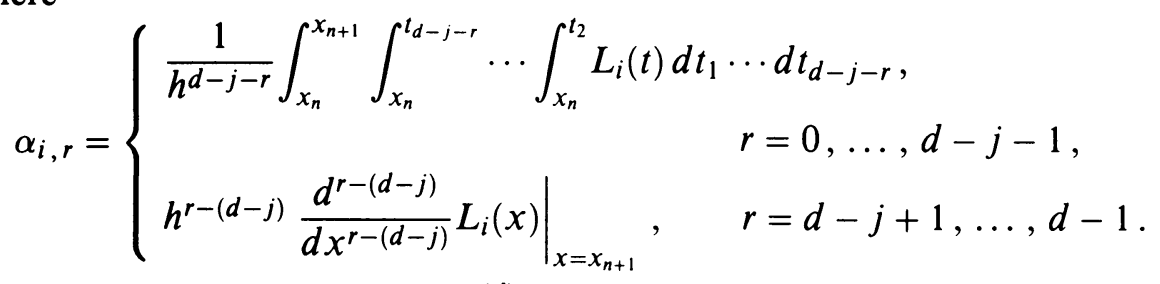

Differentiating successively for $y_{n+1}^{(d)}$, which is needed to satisfy (3.1), we obtain

$$
\sum_{i=0}^{k} \alpha_{i, d} y_{n+1-i}^{(d-j)}=h^{j} f_{n+1}
$$


where $f_{n+1}=f\left(x_{n+1}, Y_{n+1}\right)$. Replacing $Y_{n+1}$ in terms of the derivative $y_{n+1}^{(d-j)}$ given in (3.2), we then solve (3.3) for $y_{n+1}^{(d-j)}$, from which the other derivatives can then be obtained. Our discussion will be based on a generalized version of the equation (3.3), viz.,

$$
\sum_{i=0}^{k} \alpha_{i, d} y_{n+1-i}^{(d-j)}=h^{j} \sum_{i=0}^{k} \beta_{i} f_{n+1-i},
$$

where $\left|\alpha_{k, d}\right|+\left|\beta_{k}\right|>0$ and $\left|\alpha_{0, d}\right| \neq 0$. Hence, for $\beta_{0}=1$ and $\beta_{i}=0, i=$ $1, \ldots, k$, we obtain (3.3). In the implementation of the method, the difference equation (3.4) is solved for $y_{n+1}^{(d-j)}$ with the help of (3.2). With the linear multistep method (3.4) we associate the polynomials $\rho(z)=\sum_{i=0}^{k} \alpha_{i, d} z^{k-i}$ and $\sigma(z)=\sum_{i=0}^{k} \beta_{i} z^{k-i}$. Convergence for the method above is defined in the usual way.

Definition 1. The multistep method (3.2), (3.4) is said to be $y^{(t)}$-convergent if for any function $f(x, Y)$ for which a unique solution to (1.1) exists one has

$$
\lim _{\substack{h \rightarrow 0 \\ n \rightarrow \infty}} y_{n}^{(t)}=y^{(t)}(x)
$$

where $n=(x-a) / h$ for any $x \in[a, b]$, and for all solutions $\left\{y_{n}^{(t)}\right\}$ of $(3.2)$, (3.4) with starting values $y_{\mu}^{(t)}=\eta_{\mu}^{(t)}(h)$ satisfying the conditions $\lim _{h \rightarrow 0} \eta_{\mu}^{(t)}(h)=$ $\eta_{0}^{(t)}, \mu=0,1, \ldots, k-1$, where

$$
y^{(t)}(\mu h)=\eta_{0}^{(t)}+\mu h \eta_{0}^{(t+1)}+\cdots+\frac{(\mu h)^{(d-t-1)}}{(d-t-1) !} \eta_{0}^{(d-1)}+R_{d}
$$

and the remainder term satisfies $R_{d}=O\left((\mu h)^{(d-t)}\right)$.

The method is said to be convergent if it is $y^{(t)}$-convergent for $t=0,1,2, \ldots$, $d-1$; that is, all the derivatives converge to the true solutions.

For both zero stability and consistency conditions for convergence, we allude only to $y^{(d-j)}$-convergence. These may not be the only conditions, as they are related only to the coefficients $\alpha_{i, d}$ of (3.4). There may need to be other conditions related to the coefficients $\alpha_{i, r}$ of (3.2).

\section{ZERO STABILITY AND ZERO STABLE METHODS}

Theorem 1. A necessary condition for $y^{(d-j)}$-convergence (hence convergence) of the linear multistep method (3.2), (3.4) is that the modulus of no zero of $\rho(\xi)$ exceed 1 and the multiplicity of any zero with unit modulus be at most $j$. (This is termed the condition of zero stability.)

Proof. The following proof is a generalization of that given in [4]. For convenience of notation, we deal first with the case $d=j$. Consider the initial value problem $y^{(j)}=0, y^{(r)}(0)=0, r=0,1, \ldots, j-1$, whose exact solution is $y(x)=0$. The difference equation (3.4) then becomes

$$
\sum_{i=0}^{k} \alpha_{i, d} y_{n+1-i}=0
$$


Since the method is convergent, we have $\lim _{h \rightarrow 0, n \rightarrow \infty} y_{n}=0$ for $x>0$. If $\xi=r e^{i \phi}$ is a root of $\rho(\xi)=0$ of multiplicity at most $j$, then $y_{n}=h^{j} r^{n} e^{i n \phi}$ defines a solution and satisfies the initial condition in Definition 1. Hence, if $y_{n}$ is convergent, it follows that $r \leq 1$.

Similarly, if $\xi$ is a root of multiplicity greater than $j$, then $y_{n}=h^{j-1 / 2} n^{j} r^{n} e^{i n \phi}$ satisfies the initial conditions. Therefore, if $y_{n}$ is convergent, we have $r<1$.

For the case $d \neq j$, we consider the initial value problem $y^{(d)}=0$ with initial conditions $y^{(r)}(0)=0, r=0,1, \ldots, d-1$, and exact solution for $y^{(d-j)}(x)=0$. This proves the theorem.

To determine for which $k$-step methods (for different values of $j$ ) the linear difference equation (3.4) is zero stable, we find the roots of the characteristic polynomial $\rho$. We proceed in the following way to obtain $\alpha_{i, d}$, the coefficients of the characteristic polynomial $\rho$, written henceforth $\alpha_{i}(k)$ to distinguish the various $k$-step methods.

The backward difference representation of the polynomial $P_{k}(x)$ is given by

$$
P_{k}(x)=\sum_{m=0}^{k}(-1)^{m}\left(\begin{array}{c}
-s \\
m
\end{array}\right) \nabla^{m} y_{n+1}^{(d-j)}, \quad s=\frac{x-x_{n+1}}{h} .
$$

Differentiating $j$ times at $x=x_{n+1}$ gives

$$
P_{k}^{(j)}\left(x_{n+1}\right)=\frac{1}{h^{j}} \sum_{m=0}^{k} \delta_{j, m} \nabla^{m} y_{n+1}^{(d-j)},
$$

where

$$
\delta_{j, m}=\left.(-1)^{m} h^{j} \frac{d^{j}}{d x^{j}}\left(\begin{array}{c}
-s \\
m
\end{array}\right)\right|_{x=x_{n+1}}=\left.(-1)^{m} \frac{d^{j}}{d s^{j}}\left(\begin{array}{c}
-s \\
m
\end{array}\right)\right|_{s=0} .
$$

In order to obtain a useful recurrence relation for $\delta_{j, m}$, the method of generating functions is used. Let

$$
D_{j}(t)=\sum_{m=0}^{\infty} \delta_{j, m} t^{m}
$$

Then

Hence,

$$
\begin{aligned}
D_{j}(t) & =\left.\sum_{m=0}^{\infty}(-t)^{m} \frac{d^{j}}{d s^{j}}\left(\begin{array}{c}
-s \\
m
\end{array}\right)\right|_{s=0}=\left.\frac{d^{j}}{d s^{j}}\left(\sum_{m=0}^{\infty}\left(\begin{array}{c}
-s \\
m
\end{array}\right)(-t)^{m}\right)\right|_{s=0} \\
& =\left.\frac{d^{j}}{d s^{j}}(1-t)^{-s}\right|_{s=0}=\left.\frac{d^{j}}{d s^{j}} e^{-s \log (1-t)}\right|_{s=0} .
\end{aligned}
$$

$$
D_{j}(t)=(-1)^{j} \log ^{j}(1-t) .
$$

For the case $j=1$, it has been proved [1] that the polynomials of degree one to six are zero stable. Here, the cases $j=2$ to $j=5$ are examined. For $j=2$, we have from (4.2),

$$
\begin{aligned}
\delta_{2,0} & +\delta_{2,1} t+\delta_{2,2} t^{2}+\cdots=\left(t+\frac{1}{2} t^{2}+\frac{1}{3} t^{3}+\cdots\right)\left(t+\frac{1}{2} t^{2}+\frac{1}{3} t^{3}+\cdots\right) \\
& =t^{2}+\left(\frac{1}{2} \cdot \frac{1}{1}+\frac{1}{2} \cdot \frac{1}{1}\right) t^{3}+\left(\frac{1}{3} \cdot \frac{1}{1}+\frac{1}{2} \cdot \frac{1}{2}+\frac{1}{3} \cdot \frac{1}{1}\right) t^{4}+\cdots
\end{aligned}
$$


Equating coefficients gives

$$
\begin{aligned}
& \delta_{2, m}=0, \quad m=0,1, \\
& \delta_{2, m}=\sum_{r=1}^{m-1} \frac{1}{m-r} \frac{1}{r}, \quad m=2,3, \ldots .
\end{aligned}
$$

For $j=3$, we have

$$
\begin{aligned}
\delta_{3,0} & +\delta_{3,1} t+\delta_{3,2} t^{2}+\delta_{3,3} t^{3}+\delta_{3,4} t^{4}+\delta_{3,5} t^{5}+\cdots \\
& =\left(t+\frac{1}{2} t^{2}+\frac{1}{3} t^{3}+\cdots\right)^{3} .
\end{aligned}
$$

Equating coefficients gives

$$
\begin{aligned}
& \delta_{3, m}=0, \quad m=0,1,2, \\
& \delta_{3, m}=\sum_{r=2}^{m-1} \frac{1}{m-r} \sum_{p=1}^{r-1} \frac{1}{r-p} \frac{1}{p}, \quad m=3,4, \ldots .
\end{aligned}
$$

Similarly, for $j=4$,

$$
\begin{aligned}
& \delta_{4, m}=0, \quad m=0,1,2,3, \\
& \delta_{4, m}=\sum_{r=3}^{m-1} \frac{1}{m-r} \sum_{p=2}^{r-1} \frac{1}{r-p} \sum_{q=1}^{p-1} \frac{1}{p-q} \frac{1}{q}, \quad m=4,5, \ldots
\end{aligned}
$$

For $j=5$,

$$
\begin{aligned}
& \delta_{5, m}=0, \quad m=0,1,2,3,4, \\
& \delta_{5, m}=\sum_{r=4}^{m-1} \frac{1}{m-r} \sum_{p=3}^{r-1} \frac{1}{r-p} \sum_{q=2}^{p-1} \frac{1}{p-q} \sum_{s=1}^{q-1} \frac{1}{q-s} \frac{1}{s}, \quad m=5,6, \ldots .
\end{aligned}
$$

Since

we get

$$
\sum_{m=0}^{k} \delta_{j, m} \nabla^{m} y_{n+1}^{(d-j)}=\sum_{m=0}^{k} \alpha_{m}(k) y_{n+1-m}^{(d-j)}
$$

$$
\alpha_{m}(k)=(-1)^{m} \sum_{r=m}^{k}\left(\begin{array}{c}
r \\
m
\end{array}\right) \delta_{j, r}
$$

This leads to the relation

$$
\alpha_{m}(k+1)=\alpha_{m}(k)+(-1)^{m}\left(\begin{array}{c}
k+1 \\
m
\end{array}\right) \delta_{j, k+1}, \quad m=0,1, \ldots, k,
$$

and $\alpha_{k+1}(k+1)=(-1)^{k+1} \delta_{j, k+1}$.

Tables of $\alpha_{m}(k)$ for $j=2,3,4$ and 5 are given below. $j=2$

\begin{tabular}{|cccccccccc|}
\hline$m$ & 0 & 1 & 2 & 3 & 4 & 5 & 6 & 7 & 8 \\
\hline$\delta_{2, m}$ & 0 & 0 & 1 & 1 & $11 / 12$ & $5 / 6$ & $137 / 180$ & $7 / 10$ & $1089 / 1680$ \\
\hline
\end{tabular}




\begin{tabular}{|r|rrrrrrrrr|}
\hline$m$ & 0 & 1 & 2 & 3 & 4 & 5 & 6 & 7 & 8 \\
\hline$\alpha_{m}(2)$ & 1 & -2 & 1 & & & & & \\
$\alpha_{m}(3)$ & 2 & -5 & 4 & -1 & & & & \\
$12 \alpha_{m}(4)$ & 35 & -104 & 114 & -56 & 11 & & & \\
$12 \alpha_{m}(5)$ & 45 & -154 & 214 & -156 & 61 & -10 & & & \\
$180 \alpha_{m}(6)$ & 812 & -3132 & 5256 & -5080 & 2970 & -972 & 137 & & \\
$180 \alpha_{m}(7)$ & 938 & -4014 & 7911 & -9490 & 7380 & -3618 & 1019 & -126 \\
$5040 \alpha_{m}(8)$ & 29531 & -138528 & 312984 & -448672 & 435330 & -284256 & 120008 & -29664 & 3267 \\
\hline
\end{tabular}

$j=3$

\begin{tabular}{|cccccccc|}
\hline$m$ & 3 & 4 & 5 & 6 & 7 & 8 & 9 \\
\hline$\delta_{3, m}$ & 1 & $3 / 2$ & $7 / 4$ & $15 / 8$ & $29 / 15$ & $469 / 240$ & $29531 / 15120$ \\
\hline
\end{tabular}

\begin{tabular}{|r|rrrrrrrr|}
\hline$m$ & 0 & 1 & 2 & 3 & 4 & 5 & 6 & 7 \\
\hline$\alpha_{m}(3)$ & 1 & -3 & 3 & -1 & & & & \\
$2 \alpha_{m}(4)$ & 5 & -18 & 24 & -14 & 3 & & & \\
$4 \alpha_{m}(5)$ & 17 & -71 & 118 & -98 & 41 & -7 & & \\
$8 \alpha_{m}(6)$ & 49 & -232 & 461 & -496 & 307 & -104 & 15 & \\
$120 \alpha_{m}(7)$ & 967 & -5104 & 11787 & -15560 & 12725 & -6432 & 1849 & -232 \\
$240 \alpha_{m}(8)$ & 2403 & -13960 & 36706 & -57384 & 58280 & -39128 & 16830 & -4216 \\
$15120 \alpha_{m}(9)$ & 180920 & -1145259 & 3375594 & -6095796 & 7392549 & -6185970 & 3540894 & -1328724 \\
\hline
\end{tabular}

\begin{tabular}{|r|c|}
\hline$m$ & 8 \\
\hline $240 \alpha_{m}(8)$ & 469 \\
$15120 \alpha_{m}(9)$ & 29532629531 \\
\hline
\end{tabular}

$j=4$

\begin{tabular}{|cccccccccccc|}
\hline$m$ & 0 & 1 & 2 & 3 & 4 & 5 & 6 & 7 & 8 & 9 & 10 \\
\hline$\delta_{4, m}$ & 0 & 0 & 0 & 0 & 1 & 2 & $17 / 6$ & $7 / 2$ & $967 / 240$ & $89 / 20$ & $4523 / 945$ \\
\hline
\end{tabular}




\begin{tabular}{|r|rrrrrr|}
\hline$m$ & 0 & 1 & 2 & 3 & 4 & 5 \\
\hline$\alpha_{m}(4)$ & 1 & -4 & 6 & -4 & 1 & \\
$\alpha_{m}(5)$ & 3 & -14 & 26 & -24 & 11 & -2 \\
$6 \alpha_{m}(6)$ & 35 & -186 & 411 & -484 & 321 & -114 \\
$6 \alpha_{m}(7)$ & 56 & -333 & 852 & -1219 & 1056 & -555 \\
$240 \alpha_{m}(8)$ & 3207 & -21056 & 61156 & -102912 & 109930 & -76352 \\
$240 \alpha_{m}(9)$ & 4275 & -30668 & 99604 & -192623 & 244498 & -210920 \\
$15120 \alpha_{m}(10)$ & 341693 & -2655764 & 9531612 & -20819409 & 30600654 & -31524696 \\
\hline
\end{tabular}

\begin{tabular}{|r|rrrrr|}
\hline$m$ & 6 & 7 & 8 & 9 & 10 \\
\hline $6 \alpha_{m}(6)$ & 17 & & & & \\
$6 \alpha_{m}(7)$ & 164 & -21 & & & \\
$240 \alpha_{m}(8)$ & 33638 & -8576 & 967 & & \\
$240 \alpha_{m}(9)$ & 123348 & -47024 & 10579 & -1068 & \\
$15120 \alpha_{m}(10)$ & 22968204 & -11646672 & 3923037 & -3923037 & 72368 \\
\hline
\end{tabular}

$$
j=5
$$

\begin{tabular}{|ccccccc|}
\hline$m$ & 5 & 6 & 7 & 8 & 9 & 10 \\
\hline$\delta_{5, m}$ & 1 & $5 / 2$ & $25 / 6$ & $35 / 6$ & $1069 / 144$ & $285 / 32$ \\
\hline
\end{tabular}

\begin{tabular}{|r|rrrrrr|}
\hline$m$ & 0 & 1 & 2 & 3 & 4 & 5 \\
\hline$\alpha_{m}(5)$ & 1 & -5 & 10 & -10 & 5 & -1 \\
$2 \alpha_{m}(6)$ & 7 & -40 & 95 & -120 & 85 & -32 \\
$6 \alpha_{m}(7)$ & 46 & -295 & 810 & -1235 & 1130 & -621 \\
$6 \alpha_{m}(8)$ & 81 & -575 & 1790 & -3195 & 3580 & -2581 \\
$144 \alpha_{m}(9)$ & 3013 & -23421 & 81444 & -166476 & 220614 & -196638 \\
$288 \alpha_{m}(10)$ & 8591 & -72492 & 278313 & -640752 & 979878 & -1039656 \\
\hline
\end{tabular}

\begin{tabular}{|r|rrrrr|}
\hline$m$ & 6 & 7 & 8 & 9 & 10 \\
\hline $2 \alpha_{m}(6)$ & 5 & & & & \\
$6 \alpha_{m}(7)$ & 190 & -25 & & & \\
$6 \alpha_{m}(8)$ & 1170 & -305 & 35 & & \\
$144 \alpha_{m}(9)$ & 117876 & -45804 & 10461 & -1069 & \\
$288 \alpha_{m}(10)$ & 774402 & -399408 & 136347 & -27788 & 2565 \\
\hline
\end{tabular}


The tables below give the roots of the polynomial for $j=2$ to $j=5$. The roots are listed by ordered triplets $(a, b, c)$, where the first letter indicates the real part of the root, the second its imaginary part and the third the modulus of the root; $(a, \pm b, c)^{m}$ will indicate that the root $a \pm i b$ is of multiplicity $m$.

$j=2$

$$
\begin{array}{ll}
\underline{k} & \text { Roots } \\
5 & (1,0,1)^{2},(0.488,0,0.488),(0.467, \pm 0.487,0.675) \\
6 & (1,0,1)^{2},(0.484, \pm 0.160,0.510),(0.444, \pm 0.672,0.806) \\
7 & (1,0,1)^{2},(0.485,0,0.485),(0.478, \pm 0.284,0.556),(0.419, \pm 0.484,0.945) \\
8 & (1,0,1)^{2},(0.484, \pm 0.113,0.497),(0.472, \pm 0.393,0.614),(0.390, \pm 1.02,1.09)
\end{array}
$$

Hence, for $j=2$, the methods are stable up to the 7-step method.

$$
j=3
$$

$$
\begin{array}{ll}
\underline{k} & \underline{\text { Roots }} \\
5 & (1,0,1)^{3},(0.588, \pm 0.256,0.642) \\
6 & (1,0,1)^{3},(0.561,0,0.561),(0.587, \pm 0.449,0.739) \\
7 & (1,0,1)^{3},(0.578, \pm 0.157,0.599),(0.616, \pm 0.652,0.897) \\
8 & (1,0,1)^{3},(0.543,0,0.543),(0.550, \pm 0.271,0.613),(0.584, \pm 0.786,0.974) \\
9 & (1,0,1)^{3},(0.538, \pm 0.109,0.548),(0.547, \pm 0.376,0.664),(0.580, \pm 0.946,1.11)
\end{array}
$$

Hence, for $j=3$, the methods are stable up to the 8-step method.

$$
j=4
$$

$$
\begin{array}{ll}
\underline{k} & \text { Roots } \\
4 & (1,0,1)^{4} \\
5 & (1,0,1)^{4},(0.667,0,0.667) \\
6 & (1,0,1)^{4},(0.657, \pm 0.232,0.697) \\
7 & (1,0,1)^{4},(0.615,0,0.615),(0.666, \pm 0.408,0.781) \\
8 & (1,0,1)^{4},(0.605, \pm 0.142,0.622),(0.678, \pm 0.566,0.883) \\
9 & (1,0,1)^{4},(0.587,0,0.587),(0.603, \pm 0.256,0.655),(0.690, \pm 0.717,0.995) \\
10 & (1,0,1)^{4},(0.580, \pm 0.104,0.590),(0.604, \pm 0.356,0.701),(0.702, \pm 0.864,1.113)
\end{array}
$$

For $j=4$, the methods are stable up to the 9-step method. Note, however, that for $k=9$ there is a pair of isolated complex roots whose moduli are very close to, but less than 1 . This makes the methods desirable only up to the eighth step. 
$j=5$

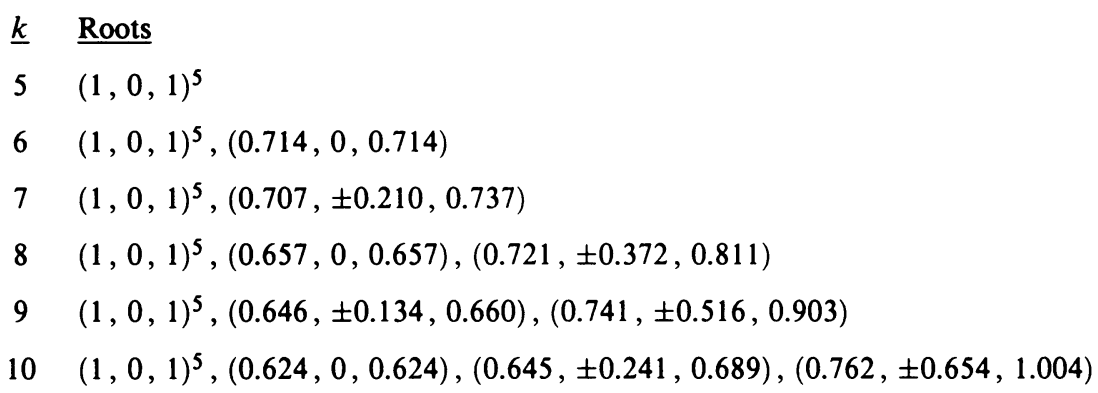

For $j=5$, the methods are stable up to the 9-step method.

\section{CONSISTENCY}

In this section, we define consistency and show it to be a necessary condition for convergence. With the difference equation (3.4), we associate the difference operator

$$
\begin{aligned}
L\left[y^{(d-j)}(x) ; h\right]= & \alpha_{k} y^{(d-j)}(x+k h)+\alpha_{k-1} y^{(d-j)}(x+(k-1) h) \\
& +\cdots+\alpha_{0} y^{(d-j)}(x) \\
& -h^{j}\left\{\beta_{k} y^{(d)}(x+k h)+\beta_{k-1} y^{(d)}(x+(k-1) h)\right. \\
& \left.+\cdots+\beta_{0} y^{(d)}(x)\right\} .
\end{aligned}
$$

Then (5.1) can be expanded in a power series of $h$ to give

$$
\begin{aligned}
L\left[y^{(d-j)}(x) ; h\right]= & c_{0} y^{(d-j)}(x)+c_{1} h y^{(d-j+1)}(x) \\
& +c_{2} h^{2} y^{(d-j+2)}(x)+\cdots+c_{q} h^{q} y^{(d-j+q)}(x)+\cdots,
\end{aligned}
$$

where the coefficients $c_{q}, q=0,1,2, \ldots$, are independent of $y^{(d-j)}(x)$.

In particular,

$$
c_{q}=\frac{1}{q !}\left(\sum_{i=0}^{k} i^{q} \alpha_{i}\right)-r\left(\sum_{i=0}^{k} i^{q-j} \beta_{i}\right),
$$

where

$$
r= \begin{cases}0, & 0 \leq q \leq j-1, \\ \frac{1}{(q-j) !}, & j \leq q .\end{cases}
$$

We define the order $p$ of the operator (5.1) to be the unique integer such that $c_{0}=c_{1}=\cdots=c_{j+p-1}=0$ but $c_{j+p} \neq 0$. If $p \geq 1$, then the method is said to be $y^{(d-j)}$-consistent.

Theorem 2. A necessary condition for the method defined in (3.2), (3.4) to be $y^{(d-j)}$-convergent (hence convergent) is that it is $y^{(d-j)}$-consistent.

To prove the above theorem, we have to show $c_{0}=c_{1}=\cdots=c_{j}=0$. Thus, in particular,

$$
c_{t}=\frac{1}{t !}\left\{0^{t} \alpha_{0}+1^{t} \alpha_{1}+2^{t} \alpha_{2}+\cdots+k^{t} \alpha_{k}\right\}=0, \quad t=0,1, \ldots, j-1,
$$


and the condition is equivalent to showing $\rho(1)=\rho^{\prime}(1)=\cdots=\rho^{(j-1)}(1)=0$ and $\rho^{(j)}(1)=j ! \sigma(1)$.

Proof of Theorem 2. Again, for simplicity of notation, we consider the case $j=d$ first.

Consider the initial value problem $y^{(j)}=0$ with $y(0)=1, y^{\prime}(0)=y^{\prime \prime}(0)=$ $\cdots=y^{(j-1)}(0)=0$ and exact solution $y(x)=1$.

The difference equation is now

$$
\alpha_{k} y_{n+k}+\alpha_{k-1} y_{n+k-1}+\cdots+\alpha_{0} y_{n}=0, \quad n=0,1,2, \ldots
$$

Assuming exact starting values, we see that the initial condition in Definition 1 is satisfied, and we must have $\lim _{h \rightarrow 0, n h=x} y_{n}=1$ for $x>0$.

Letting $n \rightarrow \infty$ in (5.3), we get

$$
\alpha_{k}+\alpha_{k-1}+\cdots+\alpha_{0}=c_{0}=0 \text {. }
$$

Next consider the problem $y^{(j)}=0$ with $y(0)=0, y^{\prime}(0)=1, y^{\prime \prime}(0)=$ $0, \ldots, y^{(j-1)}(0)=0$, whose exact solution is $y(x)=x$. Again, $y_{n}$ satisfy the initial conditions. Assuming exact starting values $y_{\mu}=\mu h, \mu=0,1, \ldots$, $k-1$, we have $y_{n}=h z_{n}$, where the sequence $\left\{z_{n}\right\}$ is also a solution to (5.3) with starting values $z_{\mu}=\mu$. Since the method is convergent,

$$
\lim _{\substack{h \rightarrow 0 \\ n h=x}} \frac{y_{n}}{x}=1 \text { for } x>0 \text {. }
$$

It follows that

$$
\lim _{n \rightarrow \infty} \frac{z_{n}}{n}=1
$$

Summing the equations

$$
\alpha_{k} z_{m+k}+\alpha_{k-1} z_{m+k-1}+\cdots+\alpha_{0} z_{m}=0 \text { for } m=0,1, \ldots, n,
$$

we obtain in view of $(5.4)$ the relation

$$
\alpha_{k} z_{n+k}+\left(\alpha_{k}+\alpha_{k-1}\right) z_{n+k-1}+\cdots+\left(\alpha_{k}+\alpha_{k-1}+\cdots+\alpha_{1}\right) z_{n+1}=-D,
$$

where $D=z_{0} \alpha_{0}+z_{1}\left(\alpha_{1}+\alpha_{0}\right)+\cdots+z_{k-1}\left(\alpha_{k-1}+\alpha_{k-2}+\cdots+\alpha_{0}\right)$ is independent of $n$. Dividing (5.6) by $n$, and letting $n \rightarrow \infty$, we obtain, using (5.5),

$$
\alpha_{k}+\left(\alpha_{k}+\alpha_{k-1}\right)+\cdots+\left(\alpha_{k}+\alpha_{k-1}+\cdots+\alpha_{1}\right)=0,
$$

that is,

$$
k \alpha_{k}+(k-1) \alpha_{k-1}+\cdots+1 \alpha_{1}=0=c_{1} .
$$

We have shown (5.2) to be true for $t=0$ and $t=1$.

We prove the general result by induction. Assume (5.2) is true for $c_{t}, 0 \leq$ $t \leq j-2$. Consider the initial value problem $y^{(j)}=0, y(0)=0, y^{\prime}(0)=$ $0, \ldots, y^{(t)}(0)=0, y^{(t+1)}(0)=1, \ldots, y^{(j-1)}(0)=0$, whose exact solution is $y=x^{t+1} /(t+1) ! ; y_{n}$ satisfies the initial conditions. Assuming exact starting values

$$
y_{\mu}=\frac{\mu^{t+1} h^{t+1}}{(t+1) !} \quad(\mu=0,1, \ldots, k-1),
$$

we obtain $y_{n}=h^{t+1} z_{n}^{t+1} /(t+1)$ !, where the sequence $\left\{z_{n}\right\}$ satisfies the difference equation

$$
k^{t} \alpha_{k} z_{m+k}+(k-1)^{t} \alpha_{k-1} z_{m+k-1}+\cdots+0^{t} \alpha_{0} z_{m}=0
$$


with starting values $z_{\mu}=\mu$. This solution is independent of $h$. For, if $\xi^{t+1}$ is a root of $\rho(s)=0$, then $\xi$ will be a root of $\sum_{i=0}^{k} i^{t} \alpha_{i} s^{i}=0$, i.e., $C z_{n}^{t+1}$ is a solution of (5.3) for some constant $C$ satisfying the initial condition in Definition 1. By convergence,

$$
\lim _{\substack{h \rightarrow 0 \\ n h=x}} \frac{y_{n}}{y(x)}=1 \text { and } \quad \lim _{n \rightarrow \infty} \frac{z_{n}}{n}=1 .
$$

Summing up (5.7) for $m=0,1, \ldots, n$, and using the fact that $c_{t}=0$ for $0 \leq t \leq j-2$, we have

$$
\begin{aligned}
& k^{t} \alpha_{k} z_{n+k}+\left\{k^{t} \alpha_{k}+(k-1)^{t} \alpha_{k-1}\right\} z_{n+k-1} \\
& +\cdots+\left\{k^{t} \alpha_{k}+(k-1)^{t} \alpha_{k-1}+\cdots+1^{t} \alpha_{1}\right\} z_{n+1}=-D,
\end{aligned}
$$

where $D=z_{1}\left(1^{t} \alpha_{1}\right)+\cdots+z_{k-1}\left\{(k-1)^{t} \alpha_{k-1}+(k-2)^{t} \alpha_{k-2}+\cdots+1^{t} \alpha_{1}\right\}$ is independent of $n$. Dividing (5.8) by $n$ and letting $n \rightarrow \infty$, we get

$$
\begin{gathered}
k^{t} \alpha_{k}+\left\{k^{t} \alpha_{k}+(k-1)^{t} \alpha_{k-1}\right\}+\cdots+\left\{k^{t} \alpha_{k}+(k-1)^{t} \alpha_{k-1}+\cdots+1^{t} \alpha_{1}\right\}=0, \\
k^{t+1} \alpha_{k}+(k-1)^{t+1} \alpha_{k-1}+\cdots+1^{t+1} \alpha_{1}=\frac{1}{(t+1) !} c_{t+1}=0,
\end{gathered}
$$

implying that $c_{t+1}=0$. Hence, (5.2) is true for all $t=0,1,2, \ldots, j-1$.

Finally, consider $y^{(j)}=j !, y(0)=y^{\prime}(0)=\cdots=y^{(j-1)}(0)=0$, with exact solution $y(x)=x^{j}$. The difference equation (3.4) now reads,

$$
\alpha_{k} y_{n+k}+\alpha_{k-1} y_{n+k-1}+\cdots+\alpha_{0} y_{n}=j ! h^{j}\left(\beta_{k}+\cdots+\beta_{0}\right) .
$$

For a convergent method, we have shown that $\rho(1)=\rho^{\prime}(1)=\cdots=\rho^{(j-1)}(1)=$ 0 , and in view of zero stability, $\rho^{(j)}(1) \neq 0$; hence

$$
k^{j} \alpha_{k}+(k-1)^{j} \alpha_{k-1}+\cdots+1^{j} \alpha_{1}=\rho^{(j)}(1)+\rho^{(j-1)}(1)+\cdots+\rho^{\prime}(1) \neq 0 .
$$

It can be verified that $y_{n}=R h^{j} n^{j}$, where

$$
R=\frac{j !\left(\beta_{k}+\beta_{k-1}+\cdots+\beta_{0}\right)}{k^{j} \alpha_{k}+(k-1)^{j} \alpha_{k-1}+\cdots+1^{j} \alpha_{1}}
$$

is a solution of (5.9) that satisfies the initial condition in Definition 1. Thus, in view of $y^{(d-j)}$-convergence of the method,

$$
R x^{j}=\lim _{\substack{h \rightarrow 0 \\ n h=x}} y_{n}=x^{j} \quad \text { for all } x>0 .
$$

It follows that $R=1$, implying $c_{j}=0$, as desired.

For the case $j \neq d$, add $(d-j)$ to the order of all the derivative values in the differential equations, with the addition of the initial values, $y(0)=$ $y^{\prime}(0)=\cdots=y^{(d-j+1)}(0)=0$, and in the discussion replace $y$ by $y^{(d-j)}$; e.g., in considering the differential equation $y^{(j)}(x)=0$ with $y(0)=0, y^{\prime}(0)=1$, $y^{\prime \prime}(0)=\cdots=y^{(j-1)}(0)=0$ with the exact solution $y(x)=x$, we obtain, if $d \neq$ $j$, that $y^{(d)}(x)=0$ with $y(0)=y^{\prime}(0)=\cdots=y^{(d-j-1)}(0)=0, y^{(d-j+1)}(0)=1$, $y^{(d-j+2)}(0)=\cdots=y^{(d-1)}(0)=0$, and the solution $y^{(d-j)}(x)=x$. 


\section{BIBLIOGRAPHY}

1. C. W. Cryer, On the instability of high order backward difference multistep methods, BIT 12 (1972), 17-25.

2. C. W. Gear, The stability of numerical methods for second-order ordinary differential equations, SIAM J. Numer. Anal. 15 (1978), 188-197.

3. G. Hall and M. B. Suleiman, Stability of Adams-type formulae for second-order ordinary differential equations, IMA J. Numer. Anal. 1 (1981), 427-438.

4. P. Henrici, Discrete variable methods in ordinary differential equations, Wiley, New York, 1962.

5. F. T. Krogh, A variable step variable order multistep method for the numerical solution of ordinary differential equations, Proc. IFIP Congr., in Inform. Process. 68 (1968), 194-199.

6. M. B. Suleiman, Convergence of the variable order and variable stepsize direct integration methods for the solution of the higher order ordinary differential equation, Pertanika 8 (1985), 59-66.

7. M. B. Suleiman and C. W. Gear, Treating a stiff single second-order ODE directly, J. Comput. Appl. Math. 27 (1989), 331-348.

8. M. B. Suleiman, Solving higher order ODEs directly by the direct integration method, Appl. Math. Comput. 33 (1989), 197-219.

9. J. M. Varah, A comparison of some numerical methods for two-point boundary value problems, Math. Comp. 28 (1974), 743-755.

Department of Mathematics, Faculty of Science and Environmental Studies, Universiti Pertanian Malaysia, 43400 UPM Serdang

E-mail address: upm@cgnet.com 